\title{
Intraoperative Vector Flow Imaging of the Heart
}

Hansen, Kristoffer Lindskov; Møller-Sørensen, Hasse; Pedersen, Mads Møller; Kjaergaard, Jesper; Nilsson, Jens Christian; Lund, Jens Teglgaard; Bachmann Nielsen, Michael; Jensen, Jørgen Arendt

Published in:

2013 IEEE International Ultrasonics Symposium Proceedings

Link to article, DOI:

10.1109/ULTSYM.2013.0445

Publication date:

2013

Document Version

Early version, also known as pre-print

Link back to DTU Orbit

Citation $(A P A)$ :

Hansen, K. L., Møller-Sørensen, H., Pedersen, M. M., Kjaergaard, J., Nilsson, J. C., Lund, J. T., Bachmann Nielsen, M., \& Jensen, J. A. (2013). Intraoperative Vector Flow Imaging of the Heart. In 2013 IEEE International Ultrasonics Symposium Proceedings (pp. 1745-1748). IEEE. https://doi.org/10.1109/ULTSYM.2013.0445

\section{General rights}

Copyright and moral rights for the publications made accessible in the public portal are retained by the authors and/or other copyright owners and it is a condition of accessing publications that users recognise and abide by the legal requirements associated with these rights.

- Users may download and print one copy of any publication from the public portal for the purpose of private study or research.

- You may not further distribute the material or use it for any profit-making activity or commercial gain

- You may freely distribute the URL identifying the publication in the public portal 


\section{Intraoperative Vector Flow Imaging of the Heart}

\author{
Kristoffer Lindskov Hansen, Hasse Møller-Sørensen, \\ Mads Møller Pedersen, Jesper Kjaergaard, Jens \\ Christian Nilsson, Jens Teglgaard Lund, Michael \\ Bachmann Nielsen \\ University Hospital of Copenhagen \\ Copenhagen, Denmark
}

\author{
Jørgen Arendt Jensen \\ Center for Fast Ultrasound \\ DTU Elektro \\ Technical University of Denmark \\ Kgs. Lyngby, Denmark
}

\begin{abstract}
The cardiac flow is complex and multidirectional, and difficult to measure with conventional Doppler ultrasound (US) methods due to the one-dimensional and angle-dependent velocity estimation. The vector velocity method Transverse Oscillation (TO) has been proposed as a solution to this. TO is implemented on a conventional US scanner (Pro Focus 2202 UltraView, BK Medical) using a linear transducer (8670, BK Medical) and can provide real-time, angle-independent vector velocity estimates of the cardiac blood flow. During cardiac surgery, epicardiac US examinations using TO were performed on three patients. Antegrade central jet and retrograde flow near the vessel wall in the ascending aorta and the pulmonary artery were seen during systole, while stable vortices were seen in the aortic sinuses and complex flow patterns were seen around the valves during diastole. In the right atrium, a stable vortex was seen during the entire heart cycle. For comparison, simultaneous measurements were obtained with conventional spectral Doppler (SD) and intravenous catheter thermodilution technique (TD). Peak systolic velocities were underestimated by $18 \%$ compared to $S D$ and cardiac output was underestimated by $16 \%$ compared to TD. This is the first time TO measurements have been obtained of cardiac flow. TO can potentially reveal new information of cardiovascular physiology and blood flow dynamics, and become a valuable tool in cardiology.
\end{abstract}

Keywords-Transverse Oscillation; vector velocity estimation; blood flow; cardiology; intraoperative ultrasound

\section{INTRODUCTION}

The cardiovascular system, composed of the heart, the valves and great vessels, confines a circuit for complex flow patterns with velocity changes and vortex formations throughout the cardiac cycle. Using both invasive and noninvasive techniques, the cardiovascular system has been investigated to understand normal and pathological flow patterns and fluid dynamics. Ultrasound (US) is the main modality for examination of the heart. However, conventional Doppler US is limited by angle-dependent and onedimensional velocity estimation that obscures the complexity of cardiac blood flow $[1,2]$.

To circumvent the angle dependency in conventional Doppler systems, a vector velocity estimator using US named Transverse Oscillation (TO) has been proposed [3]. TO has previously been tested in simulations, with flow phantoms and in-vivo [4-7]. In this study, TO implemented on a conventional US scanner provided real-time, angle-independent vector velocity estimates of cardiac blood flow with epicardiac examination on three patients undergoing cardiac surgery. The aim of the study was to investigate the feasibility of vector flow estimation using TO directly on the heart in-vivo during surgery to provide examples of vector flow measurement of different cardiac sites and to compare the measurements to conventional Doppler US and thermodilution technique (TD).

\section{MATERIALS AND METHODS}

\section{A. Patients and Setup}

The Danish National Committee on Biomedical Research Ethics approved the study. Three patients undergoing coronary bypass surgery (male: 67 years (y), female: $68 \mathrm{y}$, female: $79 \mathrm{y}$ ) with no history of valvular disease, atrial flutter or fibrillation, or septum defects entered the study after informed consent.

After standard sternotomy and before establishing coronary bypass, epicardiac scan sequences were recorded with conventional Doppler US and TO. Of the ascending aorta, additional standard Doppler measurements were obtained with transesophageal echocardiography (TEE). For one patient, pulmonary artery TD measurements were obtained and compared to volume flow derived from TO estimates.

\section{B. Transverse Oscillation}

The basis for estimation of the vector velocities with TO has been described previously [3, 4]. A conventional pulse for Doppler US is emitted and the scatterer motion is tracked along two orthogonal axes to achieve the 2-D vector velocity estimate. The motion in the axial direction is found exactly as in conventional Doppler US. The motion in the lateral direction is found by changing the sensitivity of the receiving elements to estimate the phase shift of the transverse oscillation (Fig. 1).

A conventional US scanner (Pro Focus 2202 UltraView, BK Medical) and a linear transducer (8670, BK Medical) under sterile settings were used to record epicardiac scan sequences. The TO method is implemented so real-time estimation of vector flow is achieved with a temporal resolution of $67 \mathrm{~ms}$ i.e. 16 frames/s. The angle-independent vector velocities are displayed real-time on the B-mode image as colored pixels given by a 2-D color bar defining both flow direction and velocity magnitude. To facilitate interpretation of the vector velocity estimation, small arrows are superimposed on to the color flow map. Maximum scan depth for vector flow is approximately $5 \mathrm{~cm}$ due to the transducer setup available. In this study, the ascending aorta, the pulmonary artery and the 
A

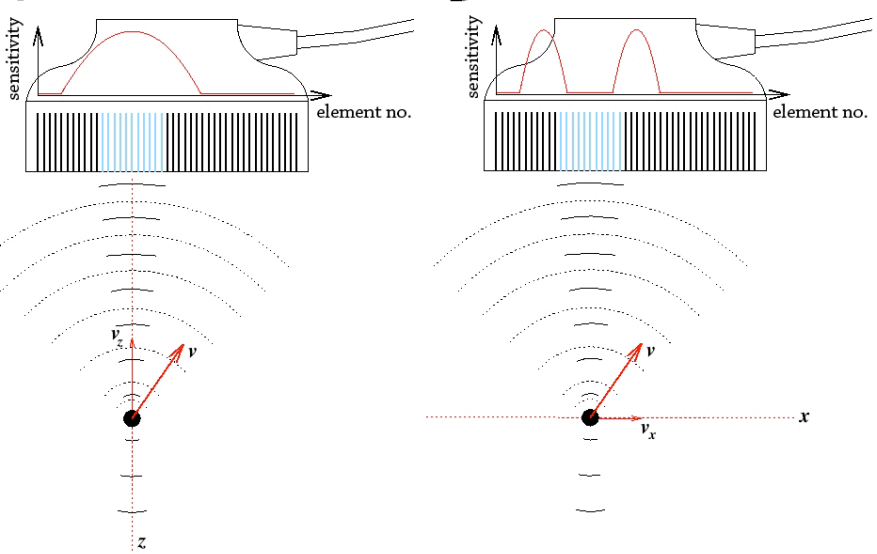

Fig. 1. The received signal from a scatterer moving along the red vector arrow $v$ is processed in two ways. In image A the axial velocity component $v_{z}$ is found by using a bell-shaped apodization function identical to conventional velocity estimation system. In image B the transverse velocity component $v_{x}$ is found by using a apodization function resembling a two-point source. The active elements in transmit are light blue, the emitted signal is represented with solid lines, and the scattered signal with dotted lines. The apodization function is shown as a red curve superimposed on the transducer.

right atrium were examined. For each scan sequence, Doppler gain, pulse repetition frequency (PRF), and wall filtering were optimized for flow imaging. Scan sequences were analyzed off-line using Matlab (Mathworks, Natick, MA, USA). Volume flow estimation was found from a line of angle-corrected estimates perpendicular to vessel boundaries by assuming circular vessel geometry and rotational symmetric flow. The measurements in comparison were recorded within a period of 10 min. Further details about the measurement setup and post processing are described by Pedersen et al. [7] and details of the volume flow calculation from vector estimates are described by Hansen et al. [6].

\section{TEE and PAC TD}

TEE scans were performed with a Philips X7-2t transducer and a Philips iE33 scanner (Philips Medical Systems, Andover, USA). Scan parameters were adjusted for flow imaging and scan sequences were stored for evaluation. Furthermore, for one patient a $7.5 \mathrm{~F}$ pulmonary artery catheter thermodilution catheter (PAC TD) (Swan-Ganz Oximetry TD catheter, Baxter Edwards Critical Care, Irvine, CA, USA) was inserted and the cardiac output measured by PAC TD was determined by the HP-Philips M1012A Cardiac Output Module (Hewlett Packard, Boeblingen, Germany).

\section{RESULTS}

\section{A. The Ascending Aorta}

The ascending aorta was examined in a long-axis and shortaxis view. Central flow downstream of the aortic valve was measured with TEE and epiaortic Doppler examination for comparison. An antegrade jet exiting the left ventricle was formed between the valve cusps in systole. For two patients the jet was skewed towards the outer curvature and for one patient towards the inner curvature. For all three patients during the entire systole, a retrograde flow along the vessel walls was seen

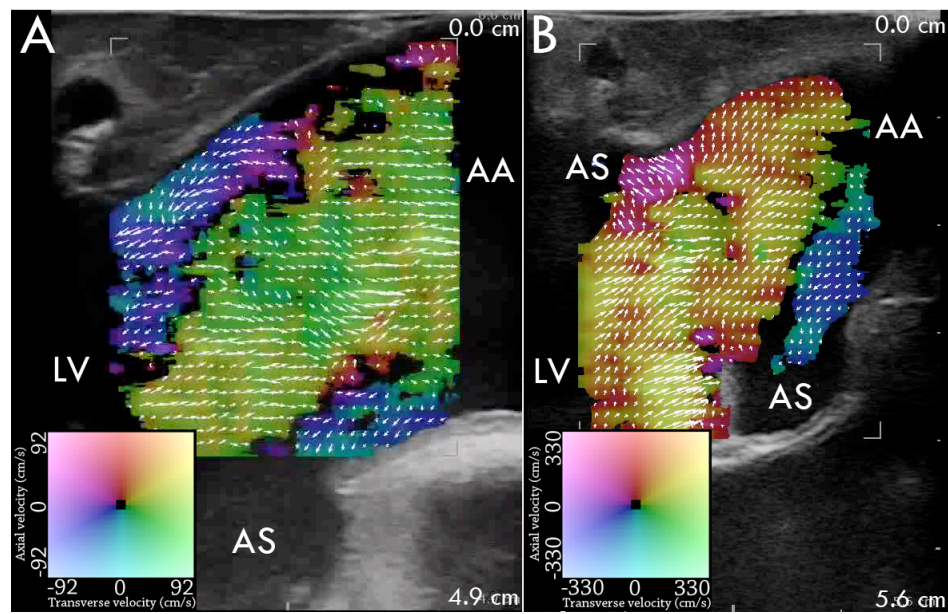

Fig. 2. The ascending aorta during systole of two patients with antegrade central flow and retrograde flow near the vessel wall. Notice the filling of the aortic sinuses in image B. LV: left ventricle. AA: ascending aorta. AS: aortic sinus.

creating vortices in the aortic sinuses (Fig. 2). In short-axis view during late systole, a well-defined clockwise secondary flow pattern was visualized in the ascending aorta (Fig. 3A). In diastole, the flow was more chaotic with a mixture of antegrade and retrograde flow, and vortices changing in place and time. However, common features during diastole were the retrograde flow being reflected on the closed valve cusps creating antegrade flow centrally and vortices formed in the aortic sinuses (Fig. 3B).

TO estimates obtained of systolic flow in the ascending aorta were compared to estimates obtained with conventional Doppler US with an epicardiac and TEE approach. For one patient, the estimation with conventional epiaortic Doppler was hindered due to pronounced cardiac movements. Maximum velocity in systole given in $\mathrm{cm} / \mathrm{s}$ in the ascending aorta was found as an average of several cardiac cycles for all three modalities. For TEE, no angle correction was performed as flow was mainly towards the transducer; for epiaortic conventional Doppler US, angle correction was employed; for TO, estimates were derived from angle-independent vector velocities, and thus, without angle correction. The averaged



Fig. 3. Image A shows the systolic clock-wise secondary flow in the ascending aorta. Image B shows the vortical retrograde flow during diastole. LV: left ventricle. AS: aortic sinus. AA: ascending aorta. 


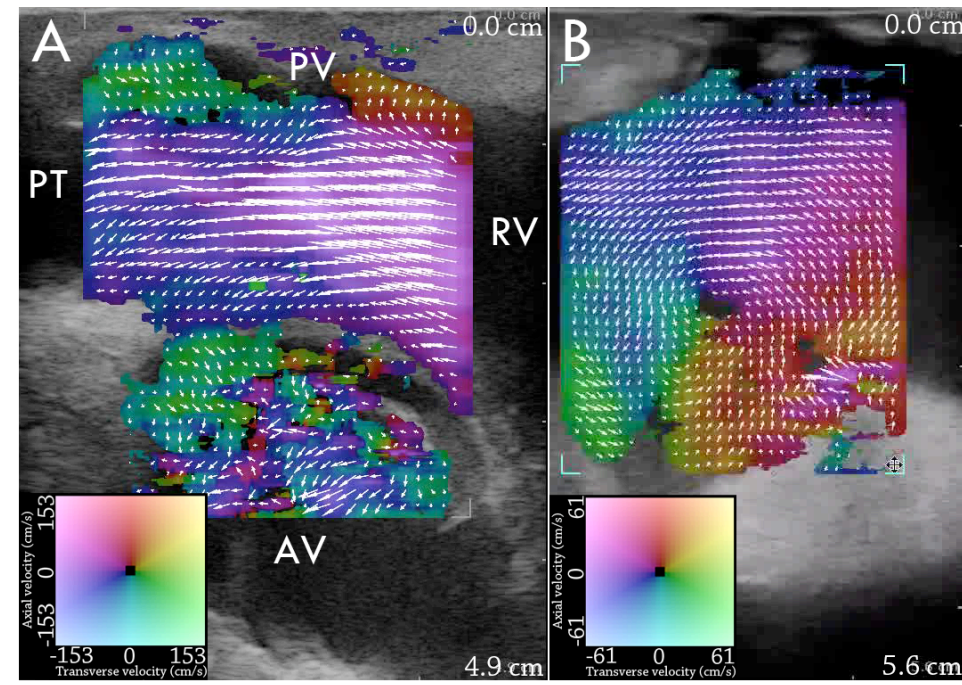

Fig. 4. Image A shows the systolic flow in the pulmonary trunk with vortical flow up- and down-stream of the valve. Image B shows the vortex in the right atrium. PT: pulmonary trunk. PV: pulmonary valve. RV: right ventricle. AV: aortic valve.

systolic velocity obtained with TO was $97 \mathrm{~cm} / \mathrm{s}$, with TEE 113 $\mathrm{cm} / \mathrm{s}$ and with epicardiac Doppler US $125 \mathrm{~cm} / \mathrm{s}$.

Furthermore, cardiac output was measured from anglecorrected vector estimates of TO and compared to PAC TD. The cardiac output and stroke volume measured with TO was $3.2 \mathrm{l} / \mathrm{min}$ and $57.1 \mathrm{ml} /$ stroke while cardiac output and stroke volume measured with PAC TD was $3.8 \mathrm{l} / \mathrm{min}$ and 63.5 $\mathrm{ml} /$ stroke, respectively.

\section{B. The Pulmonary Trunk and the Right Atrium}

The blood flow in the pulmonary trunk had a flat systolic profile when exiting the right ventricle. Retrograde vortical flow along the vessel walls was observed up- and downstream of the pulmonary valve during the entire systole (Fig. 4A). In diastole, the flow in the right ventricle was reflected on the closed valve cusps creating retrograde flow while the flow downstream of the valve primarily was antegrade created by wall-near retrograde flow reflected on the closed cusps. Finally, the right atrium was examined for one patient. A steady vortex present in systole and diastole was visualized. The vortical flow ran along the superficial wall and returned along the deep wall of the atrium redirecting inflowing blood towards the tricuspid valve (Fig. 4B).

\section{Discussion}

Other groups have previously published papers concerning angle-independent US estimations of the cardiovascular system $[8,9]$. However, to the authors knowledge this is the first paper presenting in-vivo results of real-time, quantitative US vector flow estimation of the human heart. The ascending aorta, the pulmonary trunk and the right atrium were examined with TO in three patients undergoing open-chest cardiac surgery.

\section{A. Qualitative Measurements}

During systole, a central antegrade jet was formed in the ascending aorta with retrograde flow along the vessel wall. The aortic sinuses were observed with vortices during the entire heart cycle, creating an effective closing mechanism for the aortic valve as well as providing oxygenated blood to the coronary arteries. Similar flow patterns were observed in the pulmonary artery with a systolic jet flanked by retrograde vortical flow along the vessel walls up- and downstream of the pulmonary valve.

The novel finding of retrograde flow in early systole in both the ascending aorta and pulmonary artery is probably a consequence of higher temporal and spatial resolution in the TO system compared to other modalities. TO has a spatial resolution of approx. $1 \mathrm{~mm}$ and a temporale resolution of approx. $67 \mathrm{~ms}$ while magnetic resonance imaging using phase contrast (MRI PC) has a spatial resolution of $2 \mathrm{~mm}$ and is employed by averaging over a number of heartbeats thereby removing beat-to-beat variations $[4,10]$. Conventional Doppler US has a comparable temporal and spatial resolution to the TO method but is impaired by single range-gate and onedimensional velocity estimation [1].

Secondary flow, generated by the twisting motion the myocardium, the pulsatile nature of arterial flow and the curvature of the aortic arch has been investigated with US, MRI and computational fluid dynamics [11] as well as vector flow US techniques [12]. TO revealed for two examined patients a systolic clockwise secondary flow in ascending aorta. Also a steady vortex in the right atrium was observed in both systole and diastole running along the superficial wall towards the right ventricle and returning along the deep wall. This has previously been shown with MRI by Kilner et al. who argued that vortical flow formations in the cavities of the heart conserve energy and limit flow instability [13].

\section{B. Quantitative Measurements}

The quantitative measurements of blood flow in the ascending aorta obtained with TO were compared to TEE, epiaortic conventional Doppler US and PAC TD. The peak systolic velocities were lower when measured with TO compared to TEE and conventional Doppler US with a mean difference of $18 \%$. Previous validation studies have indicated that peak systolic velocities obtained with TO are underestimated in flow-rig settings and when compared to conventional spectral US [4, 7]. Udesen et al. found for transverse flow an underestimation of $10 \%$ and furthermore, showed that the aliasing according to the Nyquist theorem of the axial velocity component is six times higher than the transverse velocity component. Pedersen et al. found $8 \%$ underestimation in peak systole and $27 \%$ overestimation in end diastole, and argued that relatively low temporal resolution and wall-filter settings contributed to the bias [7].

Volume flow was underestimated with TO compared to PAC TD with a difference of $16 \%$, and $10 \%$ when calculating the stroke volume. The TO derived stroke volume estimates have previously been compared to MRI PC with a mean underestimation of $5 \%[5,6]$. The underestimation of stroke volume was partly due to underestimated velocities. However, 
the assumptions of circular geometry and rotationally symmetric flow also contributed to the bias. Hansen et al. showed, that stroke volume calculated from long-axis 2-D data had a mean variation of $24 \%$ mainly because of asymmetric flow [6]. Furthermore, as a consequence of cardiac motion, it was difficult to secure scans exactly along the centerline of the vessel resulting in reduced lumen, and as epicardiac scans are performed directly on a beating heart, slow moving scatterers along vessel boundaries were obscured due to wall filtering. Finally, it has been shown that stroke volume is a more stable variable to measure than cardiac output [14]. For one patient the difference of cardiac output was $16 \%$, while it was $10 \%$ for stroke volume, thus indicating a certain variation in flow and heart rate.

\section{Limitations of the Study}

The study using the TO method for epicardiac blood flow estimation was limited by the transducer setup as the TO setup available has a maximum scan depth of $5 \mathrm{~cm}$ using a $5 \mathrm{MHz}$ linear transducer, which implies that whole heart examination is not possible. The TO method has been investigated on phased-array transducer setup in simulations and in flow-rig but phased-array transducer setup is not yet implemented on a conventional scanner [15]. To circumvent the scan depth limitation, the TO scans were in this study done directly on the heart. For one patient the heart moved to such an extent that epiaortic measurements were impossible. It was seen in all three examined patients for some heart beats that the axial velocity component aliased in peak systole. This aliasing was difficult to recognize during real-time scanning as the large transverse velocity component was non-aliased and therefore the overall direction mainly unaltered. Only 2-D scan plane was achieved by TO so out-of-plane scatterer movement was not visualized. Even though all measurements in comparison were achieved within 10 min some variation must be expected. This can also explain the difference between the TEE and epiaortic measurements and probably also a part of the difference between estimates of the reference methods and TO. The inherent inaccuracies in the reference methods were probably also important confounders [16].

\section{CONCLUSION}

This is the first study to visualize and estimate cardiac blood flow with the TO method, a US vector velocity estimator. TO is an angle-independent and real-time blood vector velocity method with the ability to visualize complex flow patterns and has in this study been used for intraoperative epicardiac blood flow estimations. In this study new insight in to cardiac blood flow has been provided and it has been shown that quantitative measurements of cardiac flow can be obtained from vector velocities. The TO technique may in the future become an important tool for understanding cardiac fluid dynamics, for cardiac blood flow measurements and in cardiovascular disease assessment.

\section{REFERENCES}

[1] K. L. Hansen, "In-vivo studies of new vector velocity and adaptive spectral estimators in medical ultrasound," Dan Med Bull, vol. 57, pp. 123, May 2010.

[2] J. A. Jensen, Estimation of Blood velocities using ultrasound: A signal processing approach. New York: Cambridge University Press, 1996.

[3] J. A. Jensen and P. Munk, "A new method for estimation of velocity vectors," IEEE Trans Ultrason Ferroelec Freq Contr, vol. 45, pp. 837$851,1998$.

[4] J. Udesen and J. A. Jensen, "Investigation of transverse oscillation method," IEEE Trans Ultrason Ferroelec Freq Contr, vol. 53, pp. 959971, 2006.

[5] K. L. Hansen, J. Udesen, N. Oddershede, L. Henze, C. Thomsen, J. A. Jensen, and M. B. Nielsen, "In vivo comparison of three ultrasound vector velocity techniques to MR phase contrast angiography," ultrasonics, vol. 49, pp. 659-67, Dec 2009.

[6] K. L. Hansen, J. Udesen, C. Thomsen, J. A. Jensen, and M. B. Nielsen, "In vivo validation of a blood vector velocity estimator with MR angiography," IEEE Trans Ultrason Ferroelec Freq Contr, vol. 56, pp. 91-100, 2009.

[7] M. M. Pedersen, M. J. Pihl, P. Haugaard, J. M. Hansen, K. L. Hansen, M. B. Nielsen, and J. A. Jensen, "Comparison of real-time in vivo spectral and vector velocity estimation," Ultrasound Med Biol, vol. 38, pp. 145-51, Jan 2012.

[8] L. Lovstakken, S. A. Nyrnes, B. O. Haugen, and H. Torp, "Angleindependent quantification of complex flow patterns in congenital heart disease," Proc IEEE Ultrason Symp, pp. 1246-1249, 2012.

[9] S. A. Nyrnes, L. Lovstakken, H. Torp, and B. O. Haugen, "Blood flow imaging-a new angle-independent ultrasound modality for the visualization of flow in atrial septal defects in children," Echocardiography., vol. 24, pp. 975-981, 2007.

[10] M. Markl, P. J. Kilner, and T. Ebbers, "Comprehensive 4D velocity mapping of the heart and great vessels by cardiovascular magnetic resonance," J Cardiovasc Magn Reson, vol. 13, p. 7, 2011.

[11] T. W. Koh, K. H. Parker, M. Kon, and J. R. Pepper, "Changes in aortic rotational flow during cardiopulmonary bypass studied by transesophageal echocardiography and magnetic resonance velocity imaging: a potential mechanism for atheroembolism during cardiopulmonary bypass," Heart Vessels, vol. 16, pp. 1-8, Dec 2001.

[12] K. L. Hansen, J. Udesen, F. Gran, J. A. Jensen, and M. Bachmann Nielsen, "In-vivo examples of flow patterns with the fast vector velocity ultrasound method," Ultraschall Med, vol. 30, pp. 471-7, Oct 2009.

[13] P. J. Kilner, G. Z. Yang, and D. N. Firmin, "Morphodynamics of flow through sinuous curvatures of the heart," Biorheology, vol. 39, pp. 409$17,2002$.

[14] M. B. Higginbotham, K. G. Morris, S. Williams, P. A. McHale, R. E. Coleman, and F. R. Cobb, "Regulation of Stroke Volume during Submaximal and Maximal Upright Exercise in Normal Man," Circ Res, vol. 58, pp. 281-291, 1986.

[15] M. J. Pihl, J. Marcher, and J. A. Jensen, "Phased-array vector velocity estimation using transverse oscillation," IEEE Trans Ultrason Ferroelec Freq Contr, vol. 59, pp. 2662-2675, 2012.

[16] P. R. Hoskins, "A review of the measurement of blood velocity and related quantities using Doppler ultrasound," Proc Inst Mech Eng H, vol. 213, pp. 391-400, 1999. 